\title{
Le harcèlement scolaire des minorités LGBTIQ
}

\author{
Arnaud Alessandrin ${ }^{1}$, Johanna Dagorn ${ }^{2}$
}

Mots clés : minorités de genre, école, violences.

\section{Introduction}

Le 8 mai 2018, Le premier ministre et la secrétaire d'Etat aux droits des femmes ont émis le souhait qu'il y ait, dans tous les établissements scolaires, des référent.e.s égalités fillesgarçons. Au-delà de la présence, par académie, de chargé.e.s de mission sur cette thématique, c'est le périmètre de cette volonté politique qui interroge. Alors que les questions d'égalité filles garçons peinent à entrer pleinement dans le champ des préoccupations de nombreux établissement, comment penser une inclusion plus large des questions de genre aux autres embranchements que la seule question Fille-Garçon ? Nous pensons bien évidemment à celle des élèves LGBTIQ -Lesbiennes, gays, bissexuel.le.s, trans, intersexes, queers- que l'institution a décidément beaucoup de mal à intégrer. Pour s'en faire une idée, regardons dans le passé récent des politiques en la matière. Déjà, en juin 2013, le rapport de Michel Teychenné intitulé « Discriminations lgbt-phobes à l'école état des lieux et recommandations » insistait sur la nécessité de prendre en considération les questions de décrochage scolaire des minorités de genre et de sexualité, de déprises relationnelles, de pratiques à risque, ainsi que de santé globale de ces minorités dans le milieu scolaire. Depuis, rien, ou presque. Une campagne contre l'homophobie sous-utilisée, des chapitres bien souvent maladroits sur le genre en filière de Sciences de la Vie et de la Terre et des ABCD de l'égalité dont on soulignera qu'ils ont réussi à plus emporter d'oppositions dans la rue que de soutiens ministériels. Ne restent que des initiatives locales et un plan d'actions contre le

\footnotetext{
${ }^{1}$ Arnaud Alessandrin est sociologue à l'université de Bordeaux, chercheur associé au LACES et Co-directeur des cahiers de la LCD (avec Johanna Dagorn). Ses travaux portent sur le genre, la santé et les discriminations. II a notamment dirigé, avec Johanna Dagorn l'enquête « Femmes et déplacements » et l'enquête « Santé LGBTI » (ARESVI). Récemment, il a publié « Que faire de nos dégouts » (Eclisse, 2021) avec Brigitte Esteve Bellebeau et Rogelio Esteve et « Déprivilégier le genre » (Double Ponctuation ed.)

2 Johanna Dagorn est docteure en sciences de l'éducation de l'université de Bordeaux et chercheuse associée au LACES. Elle codirige la revue « Les cahiers de la LCD - Lutte Contre les Discriminations » (avec Arnaud Alessandrin) dont elle codirigera le premier numéro, en 2016, intitulé « La ville face aux discriminations ». Dans la même veine, elle participe à de très nombreuses recherches sur les femmes et leurs déplacements et publie de nombreux articles sur cette thématique. Egalement spécialiste des questions de genre et d'éducation,
} 
harcèlement scolaire qui cite très marginalement les questions LGBTIQ et le lancement, depuis 2021, dans de nombreuses académies (on citera entre autre Poitiers et Toulouse en 2021), d'observatoires contre les LGBTI-phobies. Dans l'attente d'une concrétisation en termes d'actions, ces outils restent pour l'instant en suspens. C'était sans compter sur la médiatisation, sur les réseaux sociaux notamment, des cas de discriminations, de harcèlements et de violences à l'encontre des minorités de genre et de sexualité. Pourtant, au-delà des cas dramatiques qui attirent l'instant d'un regard l'attention des médias, l'actualité de la recherche montre l'urgence qu'il y a à prendre en compte ces parcours. Dans ce contexte de timide lutte contre les discriminations à l'encontre des minorités de genre (et de sexualité), revenons sur un panorama des expériences discriminatoires et de harcèlements scolaires subis par ces élèves.

\section{Préalables définitionnels : harcèlement, injures et discriminations}

II faut débuter par cela : le harcèlement est une violence (Hurrelmann, 1996). II ne s'agit pas de hasard, ou d'une relation duale dans laquelle chacun aurait une part de responsabilité. II s'agit même d'un délit! C'est dire combien travailler sur le climat scolaire ressenti par les élèves LGBTIQ est un indicateur précieux de leur inscription non seulement scolaire, mais également familiale et plus largement relationnelle ainsi que de leur bien être psychique en classe comme en dehors de la classe. D’après les enquêtes de climat scolaire réalisées par Eric Debarbieux, on peut estimer qu'en moyenne, près de $10 \%$ des élèves d'un établissement sont harcelés (2015). Ce qui représente environ 3 élèves par classe. Les analyses relativisant ce phénomène sont donc à éloigner de notre analyse, d'autant plus que des facteurs à risque, comme le genre, l'apparence ou l'orientation sexuelle sont largement documentés.

Le harcèlement ne sévit jamais seul. II se couple toujours d'injures et infuse un fort sentiment de discriminations aux personnes qu'elles ciblent. Si toutes les injures sont un stigmate, elles sont aussi un avertissement. Les insultes LGBTIQphobes sont, pour le dire avec les mots de Didier Eribon, inaugurales (1999). C'est-à-dire, pour le dire autrement, que l'insulte marque l'identité de celui ou celle qui la subit du poids de l'opprobre. Dans le cas de l'homophobie comme des autres insultes, le corps cible l'est toujours doublement. Ainsi les termes «pédés " ou « gouines » sont quasi-systématiquement précédés de qualificatifs du débord, de l'impur, du trop-plein, du malsain : " gros pédé », "sale gouine ». Le rabaissement par l'injure seule ne suffit donc pas.

Ce qui se sédimente dans les subjectivités des jeunes LGBTIQ, et plus précisément des minorités de genre, c'est le sentiment de honte, de violence, d'illégitimité. "Pourquoi moi ? » résume si bien le sociologue François Dubet face aux expériences discriminatoires. Toutes les minorités de genre ou de sexualité n'ont bien évidemment pas la même expérience scolaire, la même expérience du coming out et de la relation aux pair.e.s, mais tou.te.s expérimentent la menace de la sanction, de la mise à l'écart, du retrait voire pire la potentialité des coups. 
Cette menace et son application dans bien des cas inaugurent un sentiment de discrimination durablement ancré. A cet égard, il ne faut pas comprendre la discrimination uniquement du point de vue du droit, du refus de service par exemple, car à l'école rares sont les cas qui prennent cette forme (sinon pour les enseignant.e.s et les professionnel.le.s LGBTIQ de I'Education Nationale). II s'agit plutôt d'un sentiment, d'une émotion, une confrontation avec le fait d'être " traité différemment », " mis à part », " suspecté ». La suspicion, voilà qui est le propre des minorisé.e.s: ont-ils vraiment tout fait pour s'intégrer? Ne méritent-ils pas, au fond, ce qui leur arrive. En l'absence de politique de reconnaissance, ce régime à l'égard des minorités LGBTIQ se poursuit donc.

\section{Les LGB à l'école du genre}

On pourra faire lecomstat, si I'on se réfère à des " cas » médiatisés ou si l'on regarde autour de nous, plus biographiquement en sorte, que l'expérience scolaire des LGBTIQ est marquée par un nombre d'écueils qui les éloignent d'une participation scolaire pleine et entière. Et pourtant, la scolarité et la santé des mineur.e.s LGBTIQ scolarisé.e.s apparaît tardivement dans le champ de la recherche académique française, sous l'impulsion d'études qualitatives d'abord (Ayral, 2014 ; Pasquier, 2017) qui discutent des questions d'égalité avant d'entrer plus directement du côté des questions LGBT. Plus rarement, ce recherches ont également pu être quantitatives (Dagorn et Alessandrin, 2015) alors que des études similaires sont davantage développées en Suisse (Dayer, 2014) ou au Québec (Pullen-Sansfaçon et Meyer 2018 ; Richard et Chamberland, 2016) par exemple. C'est face à ce constat qu'une équipe de recherche pluridisciplinaire a tenté de dessiner plus précisment les contours de l'expérience des personnes (et des jeunes) LGBTIQ, dans une recherche ${ }^{33}$ par questionnaire et par entretiens. Les recherches qualitatives sur la question du vécu des minorités sexuelles scolarisées ont montrées avec précision un continuum net entre le sexisme et les homophobies (Joing-Maroye et Debardieux, 2014). L'enquête susmentionée montre par exemple que $50 \%$ des jeunes homosexuel.le.s ont ressenti des discriminations durant leur parcours scolaire. Les injures sont fréquemment citées mais les violences, intimidations, harcèlements et cyberharcèlements reviennent également. Plus encore, il apparaît qu'aux côtés des injures et du harcèlement, c'est la possibilité même de l'être qui marque les subjectivités de ces jeunes.

On devra néanmoins signifier une différence statistique entre le collègue et le lycée puisque ces faite apparaissent plus dans le premier que le second (respectivement $73 \%$ et $57 \%$ des jeunes LGB s'y sont sentis " (plutôt) pas bien »). Parmi celles et ceux qui parlent de ces évènements à des proches, rares sont les cas où les adultes encadrants apparaissent comme des ressources (on entend par " adultes encadrants l'ensemble des adultes présents dans l'établissement). Plus précisément, moins de $10 \%$ des jeunes gays, lesbiennes ou bissexuel.le.s privilégient l'équipe encadrante pour évoquer leurs expériences de violences. Si « en parler » nécessite de savoir " de quoi » parler, avec quels mots, cela nécessite aussi un climat de

\footnotetext{
${ }^{3}$ Dagorn, Johanna, et Arnaud Alessandrin. "La santé des élèves LGBTI », L'école des parents, vol. 627, no. 2, 2018, pp. 28-29.
} 
confiance. Or, sur cette question, 7,1\% des répondant.e.s de l'enquête se déclarent par exemple "pansexuel.le.s » (principalement des moins de 25ans) : mais à qui prononcer ces identités encore peu connues du grand public ? Comment faire reconnaitre ces identités ? Quelles représentations pèsent sur ces jeunes, en matière de réception incertaine notamment. En contrepartie, les réseaux sociaux et les ami.e.s sont de loin perçus comme des ressources premières...devant les parents. Loin de l'idée que les réseaux sociaux seraient uniquement des lieux d'agression, ils se présentent ici comme des lieux de soutien. II s'esquisse aussi une tendance générale à laquelle il convient d'être attentif, à savoir l'augmentation tendancielle des identifications par soi (" queer " « pansexuel.le.s ») ne répond pas toujours aux grammaires connues et maitrisées par les adultes en milieu scolaire ( " gays » ou « homosexuels »). Ce différentiel a pour principale conséquence le sentiment, de la part de ces mêmes mineur.e.s, d'être nié.e.s, incompris.e.s. De ne pas pouvoir, en creux, être écouté. II en découle une méfiance : à quoi bon en parler?

Enfin, des conséquences en termes de santé doivent aussi être soulignée car les discriminations, injures, incompréhensions et tabou ne sont jamais en lévitation: ils retombent sur les subjectivités, les corps, des personnes concernées. II faudra aussi évoquer les cas de décrochage scolaire, d'isolement, mais aussi d'anorexie ou de boulimie comme des symptômes scolaires et sanitaires auxquels l'institution et les adultes encadrants pourraient être attentifs. Dans les nombreux témoignages recueillis, on trouve avec récurrence des éléments relatifs aux toilettes, à la cantine: dans des espaces d'interactions ou d'isolement forts, les corps LGBTIQ sont mis à l'épreuve, des regards, des coups, des bousculades, des humiliations. Ce constat n'est pas nouveau : il est repris par d'autres recherches comme celles d'Eric Debardieux (2018) sur l'oppression viriliste et les violences scolaires : « et si on s'occupait enfin des toilettes » note le chercheur, soulignant avec insistance la dimension fortement anxiogène et à risque de ces espaces peu contrôlés par l'institution. Enfin, des études plus rares en France, mais toutefois très alarmantes, mettent en lumière la sursuicidité des personnes LGBTIQ (Firdion, 2011) et, en creux, des élèves concernés !

\section{Et les éleves trans et intersexes dans tout ça ?}

Si nous avons beaucoup parlé des élèves LGBTIQ, il convient maintenant de faire un focus sur les minorités de genre et de sexe et de faire le constat que les mineur.e.s trans et intersexes sont les grand.e.s oublié.e.s des recherches comme des politiques, du moins si l'on se retourne sur un temps long (Alessandrin, 2016 ; 2017). Pourtant, les chiffres de l'enquête mentionnée ci-dessus, mettent l'accent sur l'urgence à penser aussi, et peut-être surtout, ces populations en termes de décrochage scolaire et de santé scolaire. Par exemple, plus de $82 \%$ des personnes trans et intersexes interrogées ( $N=257 ; 217$ trans et 40 intersexes) ont jugé leur expérience scolaire (plutôt) mauvaise. Ce taux, comparable aux différentes mesures du climat scolaire dans les établissements, indique une dégradation considérable des expériences vécues par ces jeunes. Si l'on regarde le verbatim de cette enquête, c'est-à-dire les témoignages laissés dans le questionnaire, nous remarquons que les jeunes trans et les jeunes intersexes témoignent de trois ruptures précises : une absence de réponses adaptées à leurs problèmes administratifs (usage du bon prénom, reconnaissance de l'identité de 
genre...); une forte tension relationnelle (harcèlements et violences entre pairs, incompréhension ou violence de certains parents) et enfin une psychiatrisation trop fréquente de leurs demandes de médicalisation et d'hormonothérapie par les infirmier.e.s scolaires comme par les parents.

Le psychiatre et militant trans Erik Schneider (2014) revenait sur ce traitement médical -et prioritairement médical- des mineur.e.s trans dans l'institution scolaire. Selon lui, cette " externalisation » (qu'il renvoie aussi à une "psychiatrisation») des demandes au sujet des mineur.e.s trans est le signe d'une pathologisation de la question trans et des corps non binaires. Pour le dire autrement, cet " embarras »-dans le meilleur des cas- de l'institution face à ces questions conduit les professionnel.le.s à prendre appui sur des aides externes (médecins, psychologues, psychiatres notamment), sans prise en compte des spécificités, des demandes individuelles des jeunes trans, queers et non-binaires. Si la question est médicale, alors l'institution scolaire en délègue le traitement (parfois sans un certain soulagement étant donné le peu de formation des professionnel.le.s). Dans un même temps, le psychiatre Erik Schneider note que les enquêtes Allemandes ou Américaines sur les processus d'accompagnement de ces mineur.e.s notent une amélioration de la participation scolaire nette, mais aussi une diminution du décrochage scolaire des jeunes trans ou non binaires lorsque les demandes des jeunes (notamment de changer de prénom) sont entendues, reconnues et accompagnées (Richard et Alessandrin, 2019).

Dans ce contexte, il apparait que les mineurs trans (et leurs parents) doivent donc « bricoler » leurs parcours et leurs besoin d'aides, au sein de parcours et de réseaux plus ou moins formels, mal identifiés par l'institution, ajoutant alors aux complications propres aux expériences de genre adolescentes une opacité des solutions présentes.

\section{Conclusion}

Le panorama qui vient d'être décrit n'est pas radieux. Pourtant il existe un espoir, tout droit sorti des nouvelles générations de personnes trans et non-binaires, et de certains de leurs parents, en ce que les éléments de fierté finissent pas - de plus en plus, mais non entièrement encore- recouvrir les hontes, les gênes, les tabous et les sanctions à l'égard des minorités LGBTIQ. A contrario, la psychiatrisation ou la pathologisation des demandes et des identités LGBTIQ augmente les risques en matière de santé psychique et physique. La non reconnaissance des expériences et des identités minoritaires laisse elle aussi place à une souscitoyenneté de fait. Si l'on peut espérer des formations et des sensibilisations plus nombreuses notamment au sein de l'Education Nationale, nous encourageons aussi le déploiement de nouvelles recherches. Nous savons combien l'inscription, dans la durée, des questions de genre est un enjeu majeur. Récemment, les programmes de formations et priorités ministérielles ont, en France, très vite glissés du " genre »-avec les ABCD de l'égalité notamment- (Dagorn, 2014) à "l'égalité filles-garçons», puis à la "laïcité », oubliant vite les questions propres aux minorités de genre et de sexualité. II ne s'agit bien évidemment pas de 
mettre en concurrence les luttes, mais au contraire de penser leurs agencements, de façon à ce qu'un thème n'en chasse pas un autre, en fonction des actualités ou des priorités académiques.

Bibliographie :

ALESSANDRIN Arnaud, Déprivilégier le genre, Double Ponctuation, 2020.

ALESSANDRIN Arnaud, Sociologie des transidentités, Cavalier bleu ed. 2018.

ALESSANDRIN Arnaud, "La transphobie en France : insuffisance du droit et expériences de discrimination », Cahiers du Genre, vol. 60, no. 1, 2016, pp. 193-212.

ALESSANDRIN Arnaud, "Enfants trans, quel accompagnement ? 》, Revue de Santé Scolaire et Universitaire, 7, 41, 2017, pp. 25-26

ALESSANDRIN Arnaud. " "Mineurs trans »: de l'inconvénient de ne pas être pris en compte par les politiques publiques », Agora débats/jeunesses, vol. 73, no. 2, 2016, pp. 7-20.

AYRAL Sylvie, La fabrique des garçons, PUF, 2014

CHAMBERLAND Line, RICHARD Gabrielle, "Changing the schoolclimate - A criticalexamination of the policies and strategiesadopted in the province of Quebec (Canada)", in RUSSELL Steven, HORN Stacey (dir.), Sexual Orientation, Genderldentity, and Schooling, New York, Oxford UniversityPress, 2016, pp. 194-215.

DAGORN, Johanna, et Arnaud ALESSANDRIN. " La santé des élèves LGBTI », L'école des parents, vol. 627, no. 2, 2018, pp. 28-29.

DAGORN Johanna, ALESSANDRIN Arnaud, "Être une fille, un gay, une lesbienne ou un.e trans au collège et au lycée », Le sujet dans la cité, 6, 2, 2015, pp. 140-149.

DAGORN Johanna "Les ABCD de l'égalité: un outil adapté et efficace », in Pour en finir avec la fabrique des garçons (S. AYRAL et Y. RAIBAUD dir.), MSHA, pp : 263-269, 2014

DAYER Caroline, ALESSANDRIN Arnaud, "L'expérience des minorités de genre et de sexualité à l'école ", in DUGAS E, FERREOL Gilles (dir.), Oser l'autre, Louvain-la-Neuve, EME, 2015, pp. $87-110$ 
DEBARBIEUX Eric, DAGORN Johanna, ALESSANDRIN Arnaud et GAILLARD Olivia, " Les violences sexistes à l'école : un oppression viriliste » Observatoire européen de la violence à l'école. Rapport en ligne, 2018.

ERIBON Didier, Réflexions sur la question gay, Gallimard, 1999.

FIRDION Jean-Marie, BECK Françoise, SCHILTZ Marie-Ange, "Les minorités sexuelles face au risque suicidaire $», \mathrm{BEH}, 2011, \mathrm{n}^{\circ}$ 47-48 : p. 508-510.

JOING-MAROYE Isabelle, DEBARBIEUX Éric (dir.), « Violences de genre et violences sexistes à l'école », Recherches et éducations, 8 \& 9, 2013.

PASQUIER Gael, « Danser à l'école primaire. Entre questionnement des stéréotypes de sexe et reconfiguration du système de genre », Terrains \& travaux, vol. 29, no. 2, 2016, pp. 195-216.

PULLEN SANSFACON Annie, MEYER Elisabeth, Supporting transgender and gender-creativ youth, Pater Lang 2018.

SCHNEIDER Erik, « Les transidentités et l'école : vu du Luxembourg » (entretien), Cahiers de la transidentité, vol.4, pp : 71-76, 2014 
